\title{
Féeries
}

Études sur le conte merveilleux, XVII $-\mathrm{XIXe}$ siècle

\section{Des contes des mille et une nuits à la pantoufle de Marignier (1730)}

Un exemple de l'influence de l'Orient sur le théâtre du XVIII ${ }^{\mathrm{e}}$ siècle

\section{Isabelle Degauque}

\section{(2) OpenEdition}

Journals

Édition électronique

URL : http://journals.openedition.org/feeries/323

DOI : $10.4000 /$ feeries.323

ISSN : 1957-7753

Éditeur

UGA Éditions/Université Grenoble Alpes

Édition imprimée

Date de publication : 1 octobre 2007

Pagination : 117-130

ISBN : 978-2-84310-101-4

ISSN : 1766-2842

Référence électronique

Isabelle Degauque, «Des contes des mille et une nuits à la pantoufle de Marignier (1730)», Féeries [En ligne], 4 | 2007, mis en ligne le 30 octobre 2008, consulté le 07 septembre 2020. URL : http:// journals.openedition.org/feeries/323; DOI : https://doi.org/10.4000/feeries.323

Ce document a été généré automatiquement le 7 septembre 2020.

(c) Féeries 


\title{
Des contes des mille et une nuits à la pantoufle de Marignier (1730)
}

Un exemple de l'influence de l'Orient sur le théâtre du XVIII siècle

\author{
Isabelle Degauque
}

1 MARIGNIER S'EST D'ABORD FAIT CONNAîTRE à la Foire Saint-Germain avec Argénie, pièce en trois actes jouée le 26 février 1729, "retouchée par les sieurs Pannard et Pontau, qui n'eut point de succès. La musique du sieur Gilliers fut ce qu'on y trouva de meilleur ${ }^{1}$ ". Lorsqu'il donne dans cette même Foire La Pantoufle ${ }^{2}$, le 20 mars 1730, précédée des Couplets en procès de Lesage et d'Orneval, et de la parodie du Télémaque de l'abbé Pellegrin par Lesage, c'est avec plus de réussite, si l'on en croit le Dictionnaire des théâtres de Paris : Marignier aurait composé sa pièce à l'occasion du «mariage du sieur Thévenard, célèbre acteur de l'Académie royale de Musique ", sujet qui " a fort contribué à son succès ${ }^{3}$ ». Reprise le 28 août 1738 à la Foire Saint-Laurent, La Pantoufle est cette fois précédée de la Muse Pantomime de Pannard et suivie du Ballet de l'Amour et de l'Innocence. En revanche, la troisième pièce connue de Marignier, Cydippe, est mal reçue : représentée à la Foire Saint-Germain le 20 février 1731, elle est précédée d'un prologue du même auteur sur la chute inopinée de L'Esclavage de Psyché de Pannard et Fagan (donné à l'ouverture de la Foire), ainsi que de La Fausse Ridicule des mêmes auteurs. Les frères Parfaict en commentent ainsi l'échec : « les auteurs de L'Esclavage de Psyché ont à leur tour été bien vengés, car la pièce de Cydippe eut encore moins de succès, et disparut au bout de douze jours pour faire place à des nouveautés ${ }^{4}$.

2 De La Pantoufle, les frères Parfaict donnent un résumé détaillé dans le Dictionnaire des théâtres de Paris :

Le sultan Soliman a trouvé par hasard dans les jardins du sérail une pantoufle, sans connaître la personne à qui elle appartient, il en devient épris avec tant de violence que, pour la trouver, il fait essayer la pantoufle premièrement à toutes les femmes et les filles du sérail, et ensuite à toutes ses sujettes. Comme cette chaussure n'a pu convenir à aucune d'elles, le sultan a pris la résolution d'envoyer Pierrot, son confident, chercher ce qu'il demande dans les pays étrangers, et surtout en France. Pierrot, de retour de son long voyage, où il n'a rien fait, rapporte la Pantoufle en présence de Roxane, jeune sultane qui est entrée au sérail depuis son départ : elle 
reconnaît cette pantoufle pour la même qui lui a été enlevée par un aigle à Athènes, un jour qu'elle était au bain. Le sultan, charmé de cette heureuse rencontre, l'épouse et cède à son frère Haly [sic] la sultane Amine, dont il commençait à devenir amoureux. Suit un divertissement et un vaudeville ${ }^{5}$.

Par son thème (les amours contrariées du sultan) et son cadre (le harem), la pièce de Marignier témoigne à sa manière de l'influence durable des Contes des Mille et Une Nuits, après sa traduction en français à partir de 1704, sur le théâtre du XVIII siècle. Il ne s'agit pas d'un calque des Contes ni d'une réécriture, mais d'un témoignage d'imprégnation culturelle : sans doute frappé, comme d'autres lecteurs, par la cruauté du roi Shahriyar, dont seules les histoires de Schéhérazade peuvent différer les effets, Marignier va construire son propre personnage de sultan selon des critères devenus peu à peu archétypaux. Une comparaison avec d'autres pièces permet aisément de mesurer la solidité de ce véritable topos de la littérature des Lumières. Mais l'auteur de La Pantoufle ne s'avère pas seulement marqué par l'attraction pour l'Orient suscitée par les Contes. Composant une pièce de circonstance pour un mariage, il s'inspire également d'un conte de fées bien connu de Perrault, Cendrillon ou la Petite Pantoufle de verre $(1697)^{6}$, et peut-être même d'une source plus ancienne : derrière l'histoire de l'aigle voleur, ne pourrait-on voir une dette envers Elien le Sophiste? Au croisement de cette double inspiration, il ajoute Pierrot qui, grâce au voyage ordonné par Soliman, joue le rôle de véritable passerelle entre l'Orient et l'Occident.

Au cœur de la vogue orientale : le personnage du sultan

4 On retrouve dans la pièce de Marignier les clichés qui circulent parmi ses contemporains sur l'Orient, et l'Empire Ottoman en particulier. Ainsi, « la scène se passe à Stamboul [sic] ", dans le sérail du sultan Soliman, dont les amours se trouvent soudainement contrariées - comme le laissait entendre le résumé des frères Parfaict par la découverte d'une ravissante pantoufle littéralement tombée du ciel. Soliman interprète ce prodige comme un signe divin : «Il semble que Mahomet lui-même ait voulu m'avertir de chercher celle qu'il destine pour combler mon bonheur. Un pareil événement n'est pas sans mystère » (sc. 12). Outre la référence au prophète de l'Islam, Marignier rappelle, à la scène 14 , la prohibition du vin pour tout musulman, par opposition au goût prononcé que lui portent les Français : dans la dernière scène, Pierrot accepte de rester au service de son maître à condition de pouvoir honorer, en buvant, « la mémoire de son pays » car il est « natif de Bourgogne » :

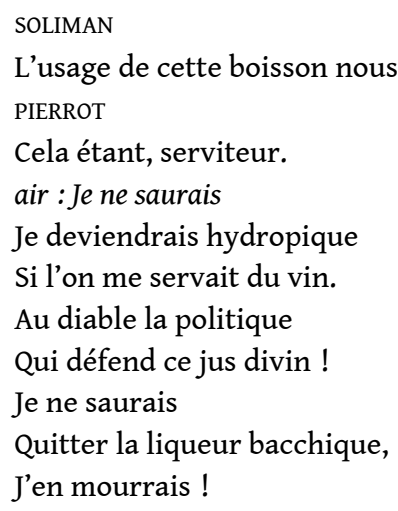

5 Ainsi, la dernière scène de La Pantoufle est prétexte à mettre en jeu l'opposition de deux cultures, respectivement incarnées par Soliman et Pierrot.

6 Marignier n'est pas un exemple isolé : d'autres dramaturges, pour les seules années 1730, s'amusent de l'attrait qu'exerce l'Orient sur l'occident, mélangent les codes et 
jouent des frictions, parfois comiques, qui résultent d'une telle confrontation. Ainsi, dans Les Enfants trouvés, ou le Sultan poli par l'amour (9 décembre 1732, ComédieItalienne), Biancolelli, Romagnesi et Riccoboni transposent à Tripoli la ville de Jérusalem où se déroulait Zaïre de Voltaire, dans la mesure où la ville libyenne véhicule au XVIII ${ }^{\mathrm{e}}$ siècle l'image d'un repaire de brigands et de corsaires, tout en conservant le cadre oriental de la tragédie prise pour cible. Hormis la mention initiale du cadre exotique, les parodistes italiens utilisent un vocabulaire dépaysant, propre à divertir leurs spectateurs : "[Je] ferais un abattis de tous les marabouts", menace Carabin (sc. 7) ; ou encore : "La mosquée est ornée, et les flambeaux s'allument ; Le muphti vous attend, déjà les parfums fument... ", invite Diaphane (sc. 9). Bien plus, les auteurs des Enfants trouvés vont reprocher à Voltaire l'inadéquation de sa représentation de l'Orient aux clichés habituels, comme l'écrit Françoise Rubellin :

La principale critique réside dans le caractère du sultan Orosmane de Voltaire, trouvé trop fade, et rebaptisé Diaphane. Dès le sous-titre, par une plaisante intertextualité avec Arlequin poli par l'amour de Marivaux (1720), le sultan de Voltaire est comparé à l'Arlequin naïf découvrant l'amour. C'est donc le manque de conformité à l'image stéréotypée du Turc que reprochent les parodistes ; le Turc en effet était alors perçu comme un être sanguinaire, comme en atteste le Dictionnaire de Furetière : "On dit aussi en voulant injurier un homme, le taxer de barbarie, de cruauté, d'irréligion, que c'est un homme inexorable, qu'il vaudrait autant avoir à faire à un Turc ». Aussi la critique est-elle récurrente dans Les Enfants trouvés : " Cadédis, pour un Turc, vous êtes honnête homme! " (sc. IV) et «Pour un Turc, avouez que je suis trop humain » (sc. XI) ; « Quoi, seigneur, de pleurer vous faites la folie » $(\mathrm{sc} . \mathrm{XV})^{7}$.

C'est donc « en creux un portrait du Turc topique, luxurieux, riche et violent, qui est dressé pour souligner le manque de ressemblance du sultan de Voltaire avec l'image attendue ${ }^{8}$ ». Or, dans La Pantoufle, Marignier reprend déjà lui aussi ces caractéristiques psychologiques pour concevoir le personnage de Soliman : il ne s'agit pas ici d'en dresser un portrait critique par rapport à un modèle existant, mais de réinvestir certains stéréotypes dans une comédie orientale conçue à la manière d'un conte, sans aucun souci vériste. Accréditant la réputation d'homme violent que serait nécessairement le Turc, Marignier fait donc se plaindre Pierrot, qui affirme à Lisette, sa bonne amie, ne pouvoir violer le serment de secret prêté à son maître, "sous peine d'empalement " (sc. 2). D'autre part, comme tout bon sultan, Soliman dispose d'un vaste harem : l'auteur de La Pantoufle y distingue trois femmes autour desquelles il va développer l'intrigue de sa pièce: l'ancienne favorite prénommée Roxane (réminiscence de l'hérö̈ne racinienne de Bajazet), désormais délaissée pour une raison qu'elle ignore; Amine, la sultane qui lui serait désormais préférée; et la perfide Atalide, dont Lisette parle comme d'un " esprit dangereux » (sc. 3). Ce trio va dessiner une géographie amoureuse qui intègre également Aly, le frère du sultan, secrètement épris d'Amine, et qui craint par là-même d'irriter Soliman. On pourrait encore ajouter à cette liste un mystérieux inconnu, accusé d'avoir séduit Roxane : ce dernier se révèlera être une esclave déguisée en homme, diligentée par Atalide pour perdre sa rivale. Le palais du sultan Soliman est en tous points conformes aux attentes des spectateurs de La Pantoufle : teinté d'érotisme et propice aux démêlés amoureux, dirigé par un maître intransigeant, jaloux de ses maîtresses et prompt à punir. C'est d'ailleurs ce que lui reproche, avec prudence, son frère, qui l'enjoint de recevoir Roxane afin qu'elle puisse se défendre : 
SOLIMAN

Je ne puis, Aly, vous accorder ce que vous me demandez; je ne verrai point la

sultane.

ALY

Air : Les Trembleurs

Une innocente victime

Que la jalousie opprime

Demande quel est son crime.

Daignez l'écouter, seigneur.

SOLIMAN

Vit-on jamais tant d'audace !

ALY

Faites-lui, du moins, la grâce,

De lui reprocher en face

La cause de son malheur.

Je suis sûr qu'elle se justifiera.

SOLIMAN

Qui peut démentir le témoignage de mes yeux ?

ALY

Souvent les apparences !

Le caractère fougueux que laisse transparaitre cet échange donne toujours à craindre pour la vie des sultanes qui pourraient décevoir le sultan, comme Lisette le fait comprendre à Amine, après que celle-ci lui a avoué aimer Aly (sc. 10) :

AIR : C'est ainsi qu'on prend [les belles]

Quand il les trouve cruelles,

Il n'en demeure pas là,

Dame, il se sert avec elles

De tous les pouvoirs qu'il a,

C'est ainsi qu'il prend les belles,

Lonlanla \&c

Redoutable, le Soliman de Largillière l'est aussi dans Ali et Zémire ${ }^{9}$, opéra-comique en un acte joué le 30 juin 1733 à la Foire Saint-Laurent. D'après la liste des personnages, Soliman n'est sultan de Déca que "par l'usurpation de son père ", à la place d'Ali, " autrefois prince de Déca » et devenu " esclave sous le nom de Zerbin ». À ces deux personnages s'ajoutent Zémire, fille de Soliman et amante d'Ali, Abelmazie, sœur d'Ali, tombée elle aussi en esclavage " sous le nom de Zaïde ", ainsi que Balkis, confidente de Zémire, Rezia, autre suivante de Zémire, et Pierrot, valet d'Ali. Des 17 scènes qui constituent cette pièce, il ne reste que des résumés, quelques airs, et le vaudeville final. Répondant elle aussi à l'engouement pour l'Orient, la pièce de Largillière partage avec La Pantoufle le cadre exotique (toute l'intrigue se tient " au sérail du sultan de Déca ») et les personnages de Soliman et d'Ali : on observe entre eux les mêmes rapports de subordination que chez Marignier, Ali déplorant dès la première scène d'être " l'esclave de l'usurpateur de sa couronne ». De plus, alors que Marignier imaginait une (illusoire) rivalité amoureuse entre les deux hommes ${ }^{10}$, ayant pour enjeu Amine, Largillière fait d'Ali le prétendant de la fille de son ennemi. D'une manière générale, l'auteur d'Ali et Zémire complique à plaisir les intrigues amoureuses : outre l'amour illicite du couple qui donne son titre à la pièce, le spectateur découvre que Balkis éprouve une violente inclination pour Ali et cherche à nuire à sa maitresse pour le lui ravir ; le sultan, lui, semble nourrir quelque secret projet envers la sœur d'Ali. Jusqu'à la dernière scène, Largillière se conforme au portrait topique du sultan inflexible, mais prend soin de préparer à la scène 14 l'heureux dénouement : « un marchand d'esclaves 
découvre au sultan la naissance d'Ali et de sa sœur. Le sultan donne des ordres secrets. Frayeur générale. On les emmène ». Ces préparatifs sont enfin révélés à la scène 17 :

On amène Ali et Abelmazie en habits royaux. Le sultan se revête d'un habit d'esclave. Ces cérémonies ne guérissent point Pierrot de ses frayeurs. Enfin, le sultan remet son sceptre et sa couronne à Ali, lui donne sa fille et prend sa sœur. Les bostangis forment le divertissement.

De La Pantoufle à Ali et Zémire, le sultan farouche se convertit finalement à la clémence : chez Marignier, il reconnaît son égarement amoureux qui lui a fait délaisser Roxane pour une chimère ; chez Largillière, informé de l'identité d'Ali et de sa sœur, il répare ses torts par un double mariage. L'intérêt de La Pantoufle est cependant d'être au carrefour des Contes des Mille et Une nuits et d'autres contes de fées.

Une autre source de La Pantoufle

$11 \mathrm{Au}$ cœur de la pièce de Marignier se trouve une mystérieuse pantoufle qui suscite l'étonnement du sultan et les pérégrinations de Pierrot. Pourtant, il faut attendre la scène 9 pour entendre de la bouche de Pierrot le récit de sa chute : Soliman, explique-til à l'attention de Lisette, avait fait emprisonner Roxane sur un soupçon d'infidélité et se préparait à donner une fête, quand " parut un aigle sur sa tête » :

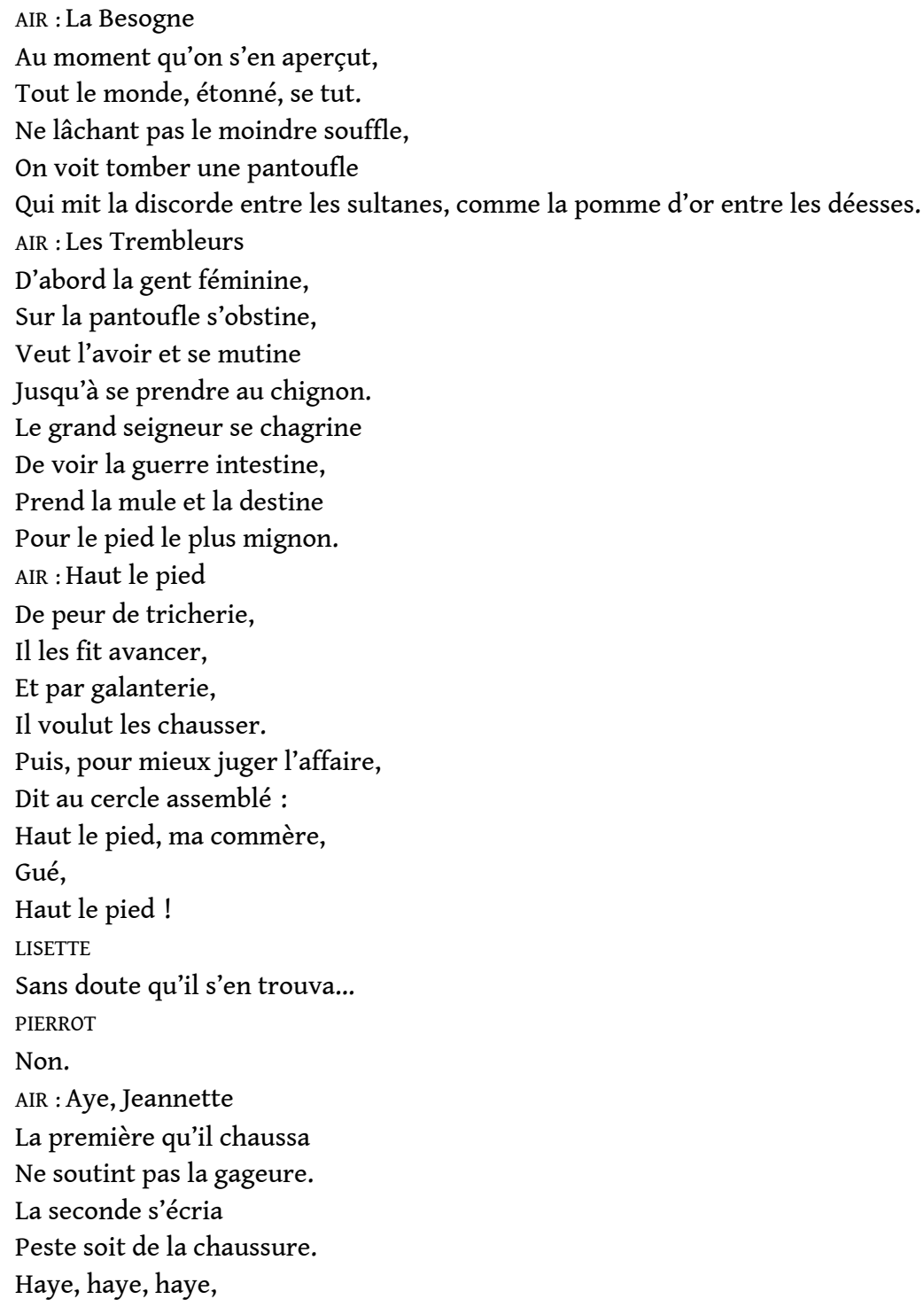


L'étroite chaussure,

Haye \&c

Elle ne put aller à personne. Et Soliman qui en était amoureux en fut charmé.

Pour rendre plus piquant son récit, Marignier choisit notamment l'air de danse « Haut le pied ", qui devient ici une invitation expresse à se soumettre à l'épreuve de la pantoufle, ou bien l'air "Aye, Jeannette ", lui aussi adéquat, puisque Marignier en exploite les onomatopées pour faire retentir les plaintes des candidates à ce concours imprévu. La concordance préside encore au choix des airs qui suivent immédiatement l'extrait : Pierrot mime le désespoir du sultan sur l'air « La sombre dondaine » :

J'ai le cœur bien malade,

Oh, oh, oh, oh,

Mon ami Pierrot,

J'ai le cœur bien malade.

En danger d'en mourir,

Pierrot, mon ami,

Tu vois mon souci.

Lassi,

Lasson, la sombre dondaine,

La sombre dondon,

Cherche le tendron,

Tâche de me guérir. (Bis)

Le comique du récit repose ici tout entier sur l'interprète Pierrot, qui doit exagérer les cris lancés sur un air de complainte galante et discréditer ainsi les émois de Soliman. Quant à Lisette, elle raille avec son amant la nouvelle passion du sultan sur un air qui en dénonce l'extravagance :

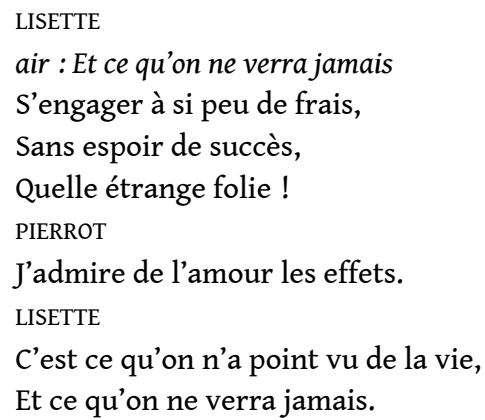

Loin d'être une fantaisie gratuite de Marignier, la pantoufle qui désespère Soliman inscrit la pièce dans une longue tradition qui a donné naissance au célèbre conte de Charles Perrault : Cendrillon ou la Petite Pantoufle de verre (1697). On retrouve d'ailleurs ici l'épreuve de la chaussure à laquelle le Prince soumet les femmes du royaume pour tenter de retrouver sa belle inconnue : "On commença à l'essayer aux Princesses, ensuite aux Duchesses, et à toute la Cour, mais inutilement ${ }^{11} »$. Toutefois, il n'est pas question chez Perrault de pantoufle tombée du ciel. Il faut remonter à une source plus ancienne pour en trouver trace : au $\mathrm{III}^{\mathrm{e}}$ siècle de notre ère, Elien rapporte, dans son Histoire variée, l'aventure de Rhodope (Yeux de rose), jeune courtisane égyptienne, à qui un aigle avait pareillement volé une pantoufle ; le pharaon Psammétique s'en entiche et promet d'épouser la femme à qui appartiendrait un soulier aussi délicat. Après maintes déconvenues, il découvre finalement son humble détentrice. Il semble que Marignier revienne à cette source pour en retenir la trame et l'adapter au nouveau contexte prisé au xvIII siècle : l'Empire Ottoman. A-t-il eu l'idée de sa pièce grâce au conte de Perrault ? Celui-ci aurait-il servi de filtre à l'histoire primitive ? 

avait quitté l'Académie royale de Musique en 1730 après une brillante carrière, a été l'occasion pour Marignier d'écrire La Pantoufle. Or, on ne peut qu'être frappé dans cette pièce par le soin apporté au récit des voyages de Pierrot en France, qui le conduisent notamment à l'Opéra. À l'occasion des noces de Thévenard, Marignier se serait donc souvenu de l'histoire de l'aigle voleur de pantoufle pour proposer une comédie sentimentale qui rend un double hommage à l'Orient si prisé, mais aussi à l'Opéra cher au dédicataire de la pièce. Et c'est grâce au personnage de Pierrot, transporté à Istanbul, que se voit assuré le passage du sérail de Soliman à la capitale française.

Pierrot au service de la satire Pantoufle, et toujours à des moments stratégiques: il ouvre la pièce par des lamentations cocasses qui vont résumer, à l'attention du spectateur, les fondements de l'intrigue :

À la fin, nous voilà de retour, qu'un esclave est à plaindre, exposé sans cesse aux caprices d'un maître impérieux, il faut qu'il obéisse sans murmurer. Soliman s'avise tout d'un coup de quitter Roxane, qui était sultane favorite, et s'entête mal à propos d'une inconnue qu'il ne verra peut-être jamais. Moi,

AIR : Naïades

Pour servir l'ardeur qui le presse,

Flatté d'une vaine promesse

De recouvrer la liberté,

J'entreprends un fâcheux voyage,

Presque à tout moment ballotté,

Par les vents, la pluie et l'orage.

Exploitant les caractéristiques de ce zanni, Marignier en fait un valet diligent mais toujours prompt à récriminer contre les caprices de son maitre, avec qui il partage sa condition d'esclave. Brave homme, Pierrot se réjouit de retrouver après quatre mois d'absence Lisette, et cette entrevue est l'occasion pour Marignier d'attiser la curiosité du public (sc. 2) : l'invitation aux embrassades est vite tempérée par Lisette qui désire apprendre ce qui a tenu si longtemps éloigné son amant ; les rebuffades essuyées ont tôt fait d'obliger Pierrot à rompre sa promesse de silence :

LISETTE

Je ne te regarderai de ma vie si tu ne contentes ma curiosité.

PIERROT

air : Eh pourquoi donc, dessus l'herbette?

Me promets-tu d'être discrète?

LISETTE

Oses-tu bien me soupçonner ?

PIERROT

$\mathrm{Va}$, cesse de te chagriner,

Tu sauras tout, Lisette,

Oui, je veux par là te donner

Preuve d'amour parfaite.

LISETTE

Apprends-moi les ordres d'un grand seigneur.

PIERROT

air : Oh! que si

Il m'a fait sortir d'ici

Chargé d'un amoureux message.

Féeries, 4 | 2007 
LISETTE

Je sais pourquoi.

PIERROT

Non, je gage.

LISETTE

Oh, que si.

PIERROT

Tu te trompes.

LISETTE

Je devine

Que c'est pour la jeune Amine.

PIERROT

Oh ! Que nanni !

LISETTE

Elle est pourtant destinée à remplacer la sultane favorite. Elle a eu toutes les peines

du monde à obtenir un délai de six mois.

PIERROT

Tout cela n'est qu'un jeu pour cacher un amour plus réel. On vient, je te rejoins dans le moment.

L'arrivée d'Atalide diffère efficacement le moment de la révélation. Dans les scènes 3 à 8, Marignier se concentre sur les affres amoureuses (jalousie d'Atalide, qui cherche à nuire à Roxane en faisant croire à Soliman qu'elle lui est infidèle ; démarches d'Ali auprès de son frère pour qu'il consente à voir cette dernière), et ce n'est que dans la longue scène 9 que l'on retrouve Pierrot, dans un nouvel entretien avec Lisette. Après le récit de l'aigle et de la pantoufle tombée du ciel, vient celui de son voyage en France : l'émissaire de Soliman retrouve ainsi " [sa] chère patrie ", dont il dresse un tableau frivole à travers le portrait d'une couturière, d'une comédienne et de deux danseuses de l'Opéra :

PIERROT

Je fis afficher ma commission avec promesse d'une riche récompense. On me mena un jour dans un endroit.

air : La Mer Rouge

En entrant dans la maison,

Je vis deux poulettes,

Joli minois, l'œil fripon,

Tailles grassouillettes.

Je leur demandai, tout doux,

Mes belles, que faites-vous ?

Je suis cou, cou, cou,

Je suis $t u, t u, t u$,

Je suis ri, ri, ri,

Je suis cou,

Je suis tu,

Je suis ri,

Je suis couturière,

Me dit la première.

Et moi, dit l'autre, je suis comédienne future.

air : Le Péril

J'ai la voix douce, harmonieuse,

Un très beau geste et du talent

Pour jouer naturellement

Les rôles d'amoureuse ${ }^{12}$.

J'en augurais favorablement. 
air : Mirlababo

Mais, hélas !, que je fus surpris,

Mirlababibobette,

Quand je vis,

Que la mule était trop étroite,

Mirlababi, serlababo,

Mirlababibobette,

Serlababorita,

Pour ce pied-là.

Enfin, j'allais à l'Opéra.

air : ô, reguingué

Je finis mes courses par là,

Et ce fut mon nec plus ultra,

Ô reguingué \&c,

En ce pays que de merveilles,

Charment les yeux et les oreilles !

Mais rien n'approche la grâce de deux jeunes élèves de Terpsichore ${ }^{13}$.

air : La Beauté

L'une fait admirer de sa taille légère

La beauté !

L'autre, en capriolant ${ }^{14}$, à peine touche à terre,

La rareté !

Et, par des entrechats, contente du parterre

La curiosité !

Le récit des pérégrinations de Pierrot trouve sa conclusion à la scène 12 (après deux scènes focalisées sur le couple Amine/Aly), dans la rencontre très attendue avec le sultan : l'infidélité que chantait Pierrot dans un dernier couplet à la scène 9 , est de nouveau célébrée sur l'air « Un sot qui veut faire l'habile » dès qu'il est question de Paris :

Ce séjour, en beautés fertiles,

N'a pu m'offrir l'objet de vos amours.

J'ai parcouru toute la ville,

Hôtels garnis, guinguettes et faubourgs,

J'ai même été pour cette créature,

Petite chaussure,

Jusqu'à l'Opéra,

Mais, ma foi, ce n'est pas là

Qu'on trouva cela.

Non content d'exploiter les clichés liés aux mœurs orientales, l'auteur de La Pantoufle s'amuse de la mauvaise réputation de la capitale française. Marignier emploie Pierrot comme le facteur dynamique de la pièce : c'est grâce à lui que le sérail s'ouvre soudain sur l'Occident, quitte à réfuter aussitôt cette percée par peur de la contamination galante. Un renversement de perspective se produit ainsi à la faveur de l'escapade de Pierrot puisque la France apparait, depuis Istanbul, comme une contrée exotique peuplée de créatures tentatrices et faciles.

21 De plus, Marignier joue des tensions inhérentes au couple traditionnel formé par le maître et son valet, et accorde à ce dernier une certaine insolence propre à faire sourire le spectateur. Les reproches formulés par Pierrot sont atténués par un préambule qui rappelle combien ce zanni est connu pour sa poltronnerie :

Je ne suis qu'un pauvre esclave.

AIR : Le Capucin

Et cependant, si sa hautesse, 
Sans songer à ma petitesse,

Voulait un moment m'écouter,

Je pourrais dire quelque chose

Dont elle pourrait profiter.

SOLIMAN

Tu n'as qu'à t'expliquer.

PIERROT

Je n'ose.

Vous vous fâcheriez.

SOLIMAN

Non, je t'en donne ma parole.

PIERROT

En vérité, seigneur, vous convient-il de vous mourir d'une passion chimérique?

Prudence et insolence se mêlent intimement chez Pierrot, et Marignier imagine ensuite une scène cocasse où Soliman s'éclipse devant son valet pour lui laisser le soin de raconter à Roxane la cause de sa froideur (sc.13). La révélation est savamment préparée : le premier air que retient Marignier autorise d'emblée le valet à railler l'aberration des émois du sultan :

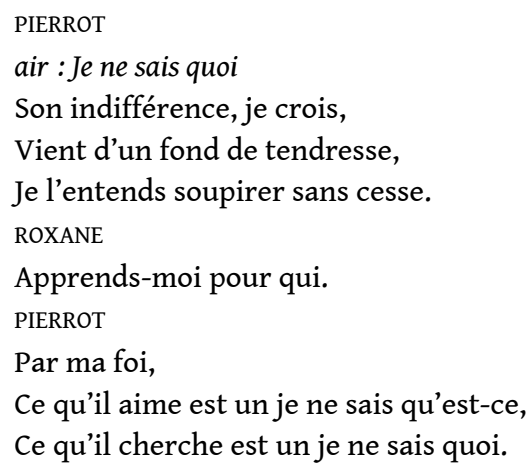

L'auteur de La Pantoufle va constamment jouer du contraste entre les paroles pleines de mystère de Pierrot et les couplets de plus en plus inquiets de Roxane. Le serviteur pousse un peu plus loin l'audace sur l'air « Le Prévôt [des marchands] » :

Sachez que c'est une beauté

Qui n'a point de réalité,

Un vain fantôme, une chimère,

Dont toute la perfection

Est dans l'espace imaginaire

Du cerveau de notre patron.

Il ignore son nom, son âge, son pays.

24 La perplexité de Roxane, à son paroxysme, est dissipée lorsque Pierrot lui présente, en un geste volontairement dramatique, « [sa] rivale ». La pantoufle est reconnue comme étant la sienne, et Soliman recouvre enfin la raison.

Ainsi, au croisement de plusieurs influences, La Pantoufle se souvient, même évasivement, des Contes des Mille et Une Nuits, mais aussi de Cendrillon ou la Petite Pantoufle de verre, et peut-être même de l'histoire de la pantoufle subtilisée par un aigle, connue dès l'Antiquité jusqu'à ce qu'elle soit popularisée en France par Charles Perrault. À cette double empreinte, s'ajoute encore la tradition de la comédie italienne et foraine à laquelle Marignier emprunte le zanni Pierrot, pour le projeter dans l'univers ottoman : faudrait-il y voir alors une invitation à lire La Pantoufle comme le mariage réussi entre la tradition italienne dell'arte et les nouvelles comédies exotiques 
propices aux intrigues amoureuses ? Enfin, la pièce de Marignier se conclut par un vaudeville dont voici le premier couplet :

Soliman, auprès de sa maîtresse,

Jouit d'un sort heureux.

Aly voit couronner sa tendresse

Par l'objet de ses feux.

Avec toi, charmante Lisette,

Je vais dans ce jour être marié !

Chacun trouve Lison, Lison, Lisette,

Chaussure à son pied.

L'air retenu par Marignier éclaire d'un autre jour La Pantoufle : si l'on se souvient que cette comédie foraine a été créée à l'occasion du mariage de Thévenard, et que l'auteur fait allusion à sa brillante carrière dans le récit de la visite de Pierrot à l'Opéra, on peut avancer l'hypothèse que c'est l'ensemble de la pièce qui fonctionne comme un hommage au célèbre acteur. Le vaudeville final révélerait que Marignier n'a composé $L a$ Pantoufle qu'en vue de la morale finale: "chacun trouve chaussure à son pied ». Allusion de circonstance à l'heureux mariage qu'il venait de contracter, mais aussi plaisanterie grivoise qu'affectionne souvent les théâtres de la Foire.

\section{NOTES}

1. Frères Parfaict, Mémoires pour servir à l'histoire des spectacles de la Foire, Paris, Briasson, 1743, t. II, p. 49.

2. Source : BnF, ms. f. fr. 9312, fo $232-247$. Pour la transcription, nous avons fait ici le choix de la modernisation d'orthographe.

3. DTP, t. IV, p. 73. N.B. : nous utilisons l'abréviation DTP pour renvoyer à l'ouvrage de Claude et François Parfaict et Godin d'Abguerbe, Dictionnaire des théâtres de Paris, rééd. Genève, Slatkine, 1967. D'après l'ouvrage que Campardon consacre à L'Académie royale de musique au xviII ${ }^{e}$ siècle, « [Thévenard] entra à l'Académie royale de Musique vers 1690 , et sept ans plus tard, en 1697, il avait complètement conquis les suffrages du public et était devenu le chanteur en vogue de l'Opéra. [...] Cet artiste quitta l'Opéra en 1730 et se retira avec une pension de retraite de 1.500 livres » (1884, t. II, p. 307).

4. DTP, t. II, p. 223.

5. DTP, t. IV, p. 72-73.

6. «Verre » est ainsi orthographié chez Perrault ; la critique s'est toutefois beaucoup interrogée à propos de cette pantoufle : peut-elle vraiment être en « verre »? ne devrait-on pas plutôt comprendre « vair » ? C'est à cette dernière interprétation que souscrivirent Balzac et Littré. Le conte imaginé en 1812 par les frères Grimm a suscité, on le sait, de semblables questions.

7. Françoise Rubellin, « L'exploitation de l'Orient dans les parodies dramatiques à la Comédie Italienne et au Théâtre de la Foire ", dans L'Oriente. Storia di una figura nelle arti occidentali (1700-2000), vol. I, Dal Settecento al Novecento, a cura di Paolo Amalfitano e Loretta Innocenti, Rome, Bulzoni Editore, 2006, p. 110.

8. Idem. 
9. Source : BnF, ms. f. fr. 9312, fo 311-314.

10.Rappel : Aly découvre à la fin de la pièce que son frère, uniquement préoccupé de savoir à qui appratenait la pantoufle affectionnée, se désintéressait en vérité d'Amine. 11. Charles Perrault, Contes, éd. Catherine Magnien, Livre de Poche, 2006, p. 268.

12. «Amoureuses » est au pluriel dans le manuscrit : pas de rime graphique avec « harmonieuse ».

13. Terpsichore, « celle qui aime les danses ", est traditionnellement représentée sous les traits d'une jeune fille enjouée qui marque la cadence des chants et de la danse chorale des sons de sa lyre. Elle fut considérée à partir du v siècle avant Jésus-Christ comme la muse de la poésie lyrique, des chœurs dramatiques et de la danse.

14. CAPRIOLE : « en termes de manège, c'est un saut que fait le cheval sans aller en avant, en sorte qu'étant en l'air, il montre les fers, et il décoche des ruades »; « [c']est aussi un saut en l'air que font les danseurs ordinaires et les sauteurs » (Furetière).

\section{RÉSUMÉS}

Pour composer une pièce de circonstance à l'occasion du mariage de l'acteur Gabriel-Vincent Thevenard, Marignier s'inspire habilement de la vogue des contes orientaux et de la notoriété de Perrault et de sa Cendrillon ou la petite pantoufle de verre. L'article montre alors comment Marignier reprend les clichés attachés au type du sultan oriental tout en déplaçant légèrement le point de vue habituel : l'intérêt de la pièce semble se focaliser sur le personnage de Pierrot, responsable de l'essayage de la pantoufle et support d'une satire qui touche surtout les mœurs occidentales. Pièce composée en hommage à un acteur de l'Opéra, La Pantoufle est une curiosité dramatique qui révèle le plaisir manifeste de l'auteur à mélanger les genres.

From the Arabian Nights to Marignier's Pantoufle (1730): an Example of Oriental Influence on XVII ${ }^{\text {th }}$ - Century Theater

In a play written to celebrate actor Gabriel Vincent Thevenard's wedding, Marignier draws his inspiration from fashionable Oriental tales, as well as from Perrault's Cendrillon or the Tiny Glass Slipper. This article shows how Marignier recycles the clichés attached to the image of the Sultan, while displacing its usual perspective : the play focuses on Pierrot, the character in charge of the fitting of the slipper and the main vehicle of a satire of Western mores. A play written as a tribute to an actor of the Opera, La Pantoufle (The Slipper) is a singular work which illustrates the obvious pleasure found by its author in hybridizing genres.

\section{AUTEUR}

\section{ISABELLE DEGAUQUE}

Université de Nantes 